\title{
Flux of biogenic carbon in oceans: size-dependent regulation by pelagic food webs
}

\author{
Louis Legendre*, Josée Michaud
}

Département de biologie, Université Laval, Québec, Québec G1K 7P4, Canada

\begin{abstract}
A central topic of modern biological oceanography is the flux of biogenic carbon (BC) towards large metazoans (i.e. renewable resources) and into deep waters (i.e. carbon sequestration. which may mitigate climate change). Two relevant characteristics of marine pelagic food webs are the turnover time of $B C(\tau)$ and the size ratio of consumers to their food particles $(\xi)$. Based on an extensive review of the literature, the present paper develops empirical equations to quantify the minimum turnover time $\left(\tau_{\min }\right)$ of $\mathrm{BC}$ incorporated in marine pelagic organisms and the residence time $\left(\tau_{\mathrm{s}}\right)$ of $\mathrm{BC}$ above depth $z_{\mathrm{s}}$ (e.g. $1000 \mathrm{~m}$ ), below which BC cannot rapidly return to the surface waters or the atmosphere. Both $\tau_{\min }$ and $\tau_{\mathrm{s}}$ are used in conjunction with $\xi$ to assess the food-web regulation of BC fluxes The paper shows that $\tau_{\text {min }}, \tau_{\mathrm{s}}$, and $\xi$ provide objective criteria for defining functional groups of organisms that are well suited for studying food-web mediated $C$ flux. As the size of organisms feeding on smaller prey increases, there is a proportional lengthening of $\tau_{\min }$ (incorporation of $B C$ in the body mass of larger organisms) and proportional shortening of $\tau_{s}$ (aggregation in faster sinking faecal pellets). The resulting increased flux towards the pools of long-lived organic $C\left(10^{-2}<\tau<10^{2}\right.$ yr $)$ and sequestered $\mathrm{BC}\left(\tau>10^{2} \mathrm{yr}\right)$ are significant for renewable resources and climate change, respectively.
\end{abstract}

KEY WORDS: Carbon flux - Ocean · Food web $\cdot$ Allometry $\cdot$ Turnover time $\cdot$ Residence time

\section{INTRODUCTION}

Two pressing global environmental issues are the depletion of marine fish stocks and the increasing concentration of atmospheric $\mathrm{CO}_{2}$, with possible resulting climate change. As a consequence, a central topic of modern biological oceanography is the flux of biogenic carbon $(\mathrm{BC})$ towards large metazoans (i.e. renewable resources) and into deep waters (i.e. carbon sequestration, which may mitigate climate change).

Legendre \& Le Fèvre (1992) defined 3 BC pools in oceans on the basis of the turnover time of BC $(\tau$ : time elapsed between the photosynthetic uptake of carbon by phytoplankton and the return of this carbon as $\mathrm{CO}_{2}$ to the surface waters or the atmosphere). The pools are short-lived organic $\mathrm{C}\left(\tau<10^{-2} \mathrm{yr} ; 3\right.$ to $\left.4 \mathrm{~d}\right)$, long-lived organic $\mathrm{C}\left(10^{-2}<\tau<10^{2} \mathrm{yr}\right)$, and sequestered $\mathrm{BC}$ $\left(\tau>10^{2} \mathrm{yr}\right.$ ). The short-lived organic $\mathrm{C}$ pool includes

•E-mail: louis.legendre@bio.ulaval.ca organisms with small $\tau$ and dissolved organic compounds; it mainly transits through microbial components of the food web. The long-lived organic $C$ pool includes renewable marine resources (e.g. fish, marine mammals) and heterotrophic bacteria involved in the breakdown of organic matter derived from large heterotrophs. The latter stresses the fact that $\tau$ is not the same as the doubling time of organisms which mediate the turnover, e.g. heterotrophic bacteria decomposing a large carcass have individual doubling times $\leq 1 \mathrm{~d}$ but they are part of a $C$ pool whose $\tau$ is much longer. Sequestered BC may take various forms, including organic remains buried in sediments (including petroleum), inorganic deposits of biological origin (e.g. calcareous ooze, coral reefs, continental limestone), refractory dissolved organic matter, and dissolved $\mathrm{CO}_{2}$ in deep waters resulting from the in situ oxidation (respiration) of organic compounds.

Fortier et al. (1994) investigated some of the mechanisms that govern the partitioning of $\mathrm{BC}$ among the 3 pools. They hypothesized that pelagic organisms 
Table 1. Symbols and abbreviations, definitions, and units of quantities used in the present paper

\begin{tabular}{|llc|}
\hline Symbol & Definition & Unit \\
\hline BC & Blogenic carbon & - \\
$D$ & Faecal pellet diameter (ESD) & $\mathrm{m}$ \\
ESD & Equivalent spherical diameter & $\mathrm{m}$ \\
$L$ & Body length & $\mathrm{m}$ \\
$M$ & Wet body mass & $\mathrm{kg}$ \\
$M C$ & Dry carbon body mass & $\mu \mathrm{g}$ \\
OLS & Ordinary least square (regression model.) & - \\
$P V$ & Faecal pellet volume & $\mu \mathrm{m}^{3}$ \\
$S$ & Sinking velocity of particles & $\mathrm{m} \mathrm{d}^{-1}$ \\
$r_{\max }$ & Maximum rate of population growth (Peters 1983) & $\mathrm{W} \mathrm{kg}^{-1}$ \\
$r_{\max }$ & Maximum rate of population growth (this paper) & $\mathrm{d}^{-1}$ \\
$Z_{5}$ & Depth below which BC becomes sequestered & $\mathrm{m}$ \\
$\xi$ & Size ratio of a consumer to its food particles & - \\
$\tau$ & Turnover time of BC & $\mathrm{d}$ \\
$\tau_{\min }$ & Minimum turnover time of BC in pelagic organisms & $\mathrm{d}$ \\
$\tau_{\mathrm{s}}$ & Residence time above $z_{\mathrm{s}}$ of sinking organic particles & $\mathrm{d}$ \\
\hline
\end{tabular}

which package small particles into larger ones generally contribute to the lengthening of $\tau$ and even to the transfor of $B C$ from a given $C$ pool to a longer-lived one. As a corollary, the lengthening of $\tau-$ and therefore the probability of shifting $\mathrm{BC}$ to a longer-lived pool-should be a direct function of the size ratio of consumers to their food particles $(\xi)$. Using data from the literature, Fortier et al. (1994) identified 4 groups of large marine planktonic microphages (salps, doliolids, appendicularians, and pteropods) as the zooplankton most efficient at re-packaging small particles into larger ones $\left(\xi>5 \times 10^{3}\right)$. For these large microphages, the study largely supported the above hypothesis and its corollary.

It follows that 2 fundamental characteristics of marine pelagic food webs, with respect to fisheries and climate change, are $\tau$ and $\xi$. The present paper develops empirical equations to quantify $\tau$ and use it in conjunction with $\xi$ to assess the food-web regulation of $\mathrm{BC}$ fluxes. The paper also shows that $\tau$ and $\xi$ provide objective criteria for defining functional groups of organisms to be used for studying food-web mediated $C$ flux in oceans.

\section{EQUATIONS AND DATA FOR QUANTIFYING $\tau$ AND $\xi$}

Several of the equations below are taken from Peters (1983), who gave allometric relationships in terms of wet body mass $\left(M_{i} \mathrm{~kg}\right)$. In the present paper, equations are stated in terms of body length $(L$; $\mathrm{m})$, because this quantity is more often reported in the literature than mass. Symbols, definitions and units of quantities used below are listed in Table 1. Peters (1983, his Appendix IIa) provided allometric relations between $M i$ and $L$ for a variety of organisms, including:

$$
\begin{aligned}
& M=0.0058 L^{1.9} \text { (phytoplankton) } \\
& M=0.08 L^{2.1} \text { (zooplankton) } \\
& M=10.6 L^{257} \text { (fish) }
\end{aligned}
$$

A general equation for pelagic organisms, ranging in size from picophytoplankton $(\leq 2 \mu \mathrm{m})$ to blue whales (up to $30 \mathrm{~m}$ ), is derived here from the $L$ and $M$ values given in Table 2 (columns 2 and 3 from the left; $M$ values in column 3 are computed using Eqs. 1-3):

$$
\begin{aligned}
M= & 6.07 L^{2.59}(\mathrm{r}=0.99, \text { ordinary least-square } \\
& \text { regression of } \log M \text { on } \log L)
\end{aligned}
$$

Table 2 shows (column 4 from the left compared to column 3) that Eq. (4) somewhat underestimates $M$ for blue whales, fish, and phytoplankton $\leq 20 \mu \mathrm{m}$, relative to Eqs. (1) \& (3), and slightly overestimates $M$ for zooplankton and large phytoplankton, relative to Eqs. (1)

\begin{tabular}{|c|c|c|c|c|}
\hline Organism & Body length (m) & Wet body mass (Eqs. $1-3)(\mathrm{kg})$ & Wet body mass (Eq. 4) (kg) & $\tau_{\min }$ (Eq. 10) (d) \\
\hline Blue whale & $3 \times 10^{1}$ & $1.5 \times 10^{5}$ & $0.4 \times 10^{5}$ & $3760^{\mathrm{a}}$ \\
\hline Fish & $2 \times 10^{0}$ & $6.3 \times 10^{1} \quad$ (Eq. 3) & $3.6 \times 10^{1}$ & 613 \\
\hline Fish & $2 \times 10^{-1}$ & $1.7 \times 10^{-1}$ (Eq. 3) & $0.9 \times 10^{-1}$ & 131. \\
\hline Euphausi.id & $2 \times 10^{-2}$ & $2.2 \times 10^{-5}$ (Eq. 2) & $24 \times 10^{-5}$ & 28 \\
\hline Copepod & $2 \times 10^{-3}$ & $1.7 \times 10^{-7}$ (Eq. 2$)$ & $6.3 \times 10^{-7}$ & 6.0 \\
\hline Tintinnid & $2 \times 10^{-4}$ & $1.4 \times 10^{-9} \quad$ (Eq. 2) & $16 \times 10^{-10}$ & 1.3 \\
\hline Microphytoplankton & $2 \times 10^{-4}$ & $5.4 \times 10^{-10}$ (Eq. 1) & $16 \times 10^{-10}$ & 1.3 \\
\hline Nanophytoplankton & $2 \times 10^{-5}$ & $6.8 \times 10^{-12}$ (Eq. 1$)$ & $4.2 \times 10^{-12}$ & 0.3 \\
\hline Picophytoplankton & $2 \times 10^{-6}$ & $8.6 \times 10^{-14}(\mathrm{Eq} .1)$ & $1.1 \times 10^{-14}$ & 0.06 \\
\hline $3760 \mathrm{~d}=10.3 \mathrm{yr}$ & & & & \\
\hline
\end{tabular}
$\&$ (2). Eq. (4) will be used below to derive $M$ from $L_{\text {, }}$ because it provides a relationship which is valid for the whole range of pelagic organisms, whereas

Table 2. Wet body mass $\left(M, 2\right.$ sets) and minimum. turnover time of $\mathrm{BC}\left(\tau_{\min }\right)$ for pelagic organisms as computed from body length $(L)$ 
Eqs. (1)-(3) are specific to phytoplankton, zooplankton and fish, respectively.

In the following sections, linear regressions will be fitted to pairs of variables that are both subject to measurement errors. It is sometimes thought that, in such cases, one must always use the major axis or the reduced major axis (geometric mean) methods (also known as Model II). McArdle (1988) and Sokal \& Rohlf (1995) explain that these methods are appropriate when the aim of the study is to derive a descriptive model from observed data, but that ordinary leastsquare (OLS) regression must be used when the objective is to obtain an expression for computing values of one variable from the values of another (i.e. forecasting, often called prediction). Since the purpose of fitting regressions below will always be to obtain expressions for calculating unknown values of one variable from known values of another, the OLS method will be systematically used.

In the present paper, distinction is made between the upper water column, where $\mathrm{BC}$ can be remineralized and the resulting $\mathrm{CO}_{2}$ can be lost to the atmosphere, and the deep waters, where $\mathrm{BC}$ becomes sequestered. The boundary between the 2 layers is defined as the depth $\left(z_{\mathrm{s}}\right.$, e.g. $\left.1000 \mathrm{~m}\right)$ below which $\mathrm{BC}$ cannot rapidly return to the surface waters or the atmosphere. The 2 global environmental issues discussed above concern 2 distinct aspects of $\tau$ in oceans. On the one hand, in the upper water column, incorporation of $\mathrm{BC}$ in the body masses of large organisms contributes to an increase in the flux towards the pool of long-lived organic $\mathrm{C}$. This is significant for renewable resources. On the other hand, export of $\mathrm{BC}$ towards deep waters augments the flux towards the pool of sequestered BC. This may be significant for climate change. The pool of long-lived organic C mainly consists of living biomass and semi-labile dissolved organic carbon (DOC), whereas the pool of sequestered BC is mainly supplied by the sinking of inert organic particles (although sinking marine snow may contain living organisms) and downward mixing of refractory DOC. The equations below address the channelling of $\mathrm{BC}$ into both longlived organisms [see 'Incorporation of carbon in the body mass of living organisms $\left.\left(T_{\text {min }}\right)^{\prime}\right]$ and fast-sinking particles [see 'Export of organic particles towards deep waters $\left.\left(\tau_{s}\right)^{\prime}\right]$. They do not consider refractory DOC, whose deep export is determined by hydrodynamics, not food-web activity.

Data used below were compiled from the literature. They concern taxa and developmental stages of marine pelagic organisms that include (in order of generally decreasing size) 4 mammals, 1 turtle, 35 fish, 3 cnidarians, 13 salps, 5 chaetognaths, 5 pteropods, 1 doliolid, 3 appendicularians, 8 euphausiids, 3 mysiids, 2 shrimps, 1 cladoceran, 81 copepods, 7 mero- planktonic larvae of benthic organisms, 17 tintinnids, 8 ciliates other than tintinnids, and 12 flagellates. For each taxon and stage, the information concerns (when available) up to 5 variables: maximum length of the organism; length of the smallest food particles efficiently collected (when the size distribution of particles exhibited several modes, the smallest-size mode was chosen; when there was no information available on collection efficiency, cited particles are the smallest among those that made up $\geq 1 \%$ of the diet of the organism); type of food; mean diameter of faecal pellets (equivalent spherical diameter, ESD; when a range of values was reported in the literature, the arithmetic mean of the 2 extreme values was computed); mean sinking velocity of faecal pellets (when a range of values was reported in the literature, the geometric mean of the 2 extreme values was computed). The choice of arithmetic and geometric means for the diameter and sinking velocity, respectively, was based on cases where the references provided both ranges and mean values. The data table is too large to be published; it is available on the InterResearch World Wide Web page (http://www.intres.com/journals/suppl/).

\section{Incorporation of carbon in the body mass of living organisms $\left(\tau_{\min }\right)$}

The minimum turnover time of $\mathrm{BC}$ incorporated in pelagic organisms $\left(\tau_{\min }\right)$ can be derived from the maximum rate of population growth $\left(r_{\max }\right)$ :

$$
\tau_{\operatorname{man}}=r_{\max }{ }^{-1}
$$

This provides an average value for individuals in the population, i.e. some individuals may turn over BC faster than $\tau_{\min }$. In Appendix VIIIc of Peters (1983), the units of $r_{\max }$ are those of production per unit biomass, i.e. power mass ${ }^{-1}\left(\mathrm{~W} \mathrm{~kg}^{-1}=\mathrm{J} \mathrm{s}^{-1} \mathrm{~kg}^{-1}\right)$. According to his Appendix Ia, the energy corresponding to $1 \mathrm{~kg}$ of wet mass is $7 \times 10^{6} \mathrm{~J}$. Hence,

$$
\begin{aligned}
& 1 \mathrm{~W} \mathrm{~kg}^{-1} \text { corresponds to } \\
& \left(1 \mathrm{~J} \mathrm{~s}^{-1}\right)\left(7 \times 10^{6} \mathrm{~J}\right)^{-1}=(1 / 7) 10^{-6} \mathrm{~s}^{-1}
\end{aligned}
$$

so that

$$
1 \mathrm{~W} \mathrm{~kg}^{-1} \text { corresponds to } 0.0123 \mathrm{~d}^{-1}
$$

In the literature, there are several equations that describe $r_{\max }$ as a function of $M$ (see Peters 1983, his Appendix VIIIc). The most general equation for pelagic organisms appears to be that of Blueweiss et al. (1978):

$$
r_{\max }\left(\mathrm{W} \mathrm{kg}^{-1}\right)=0.336 \mathrm{M}^{-0.26}
$$

where the unit of $M$ is $\mathrm{kg}$. Using Eq. (6), Eq. (7) 
becomes

$$
r_{\mathrm{F}}\left(\mathrm{d}^{-1}\right)=0.004 \mathrm{M}^{-0.26}
$$

Combining Eqs. (5) and (8) provides the following expression for $\tau_{\min }(\mathrm{d})$ :

$$
\tau_{\min }=r_{\max }{ }^{-1}=241 M^{0.26}
$$

Combining Eqs. (4) and (9) gives $\tau_{\min }$ as a function of $L$ (m)

$$
\tau_{\min }=385 L^{0.67}
$$

Table 2 (column 5) provides $\tau_{\min }$ values computed with Eq. (10) for a wide range of pelagic organisms. According to Table 2 , the average $\tau_{\min }$ for $30 \mathrm{~m}$ long blue whales is $10.3 \mathrm{yr}$, whereas the corresponding value for $2 \mu \mathrm{m}$ cyanobacteria is $1.4 \mathrm{~h}(0.06 \mathrm{~d})$. Values in Table 2 are of the appropriate order of magnitude.

The change in $\tau_{\text {min }}$ that results from smaller organisms $\left(L_{1}\right)$ being eaten by larger ones $\left(L_{2}\right)$ is computed as follows:

$$
\tau_{\min 2} / \tau_{\min 1}=\left(L_{2} / L_{1}\right)^{0.67}
$$

OI, yiven i'lad $\xi_{21}=L_{2} / L_{1}$.

$$
\tau_{\text {min2 }} / \tau_{\text {mun } 1}=\xi_{21}{ }^{0.67}
$$

Because $\tau_{\min 1} \leq \tau_{\min 2}$, the ratio is always $\geq 1$

Eq. (11) describes the lengthening of $\tau_{\min }$ that corresponds to 1 trophic transfer. In the pelagic food web, there is a large number of such transfers. Since the definition of $\tau_{\min }$ (Eq. 5) accounts for all C losses by organisms, the net result of smaller organisms being eaten by large ones, which are in turn eaten by larger ones, is to increase $\tau_{\text {mun }}$. Two contrasting cases are the microbial food web, where most $C$ fixed by phytoplankton is rapidly respired by flagellates, ciliates and heterotrophic bacteria (overall $\tau_{\text {min }}$ of a few days), and the polar herbivorous food web, where part of phytoplankton production is incorporated in the biomass of large pelagic crustaceans (copepods and krill), which are in turn eaten by marine mammals (e.g. blue whales) whose $\tau_{\text {min }}$ may reach 10 yr (Table 2). Hence, even if metabolic losses of $\mathrm{C}$ are very high throughout the food web, transfer of $\mathrm{BC}$ to progressively larger organisms increases the overall channelling of $\mathrm{BC}$ towards the pool of long-lived organic $C$.

The derivation of equations for $\tau_{\min }$ could stop here. However, Eqs. (10) \& (11) may be criticised because, as explained by Peters (1983), Eq. (7) was computed from data that had originally been analysed separately for pelagic unicells, poikilotherms, and homoiotherms. The next paragraphs show that such a level of detail can be neglected in the present approach. The original equations (Fenchel 1974) are

$$
r_{\max }\left(\mathrm{W} \mathrm{kg}^{-1}\right)=0.136 \mathrm{M}^{-0.28} \text { (unicells) }
$$

$$
\begin{aligned}
& r_{\max }\left(\mathrm{W} \mathrm{kg}^{-1}\right)=0.281 \mathrm{M}^{-0.27} \text { (poikilotherms) } \\
& r_{\max }\left(\mathrm{W} \mathrm{kg}^{-1}\right)=0.627 \mathrm{M}^{-0.27} \text { (homoiotherms) }
\end{aligned}
$$

The slopes of Eqs. (12)-(14) are very close to -0.25 and the intercepts are not very different from each other (i.e. 2-fold difference from one group of organisms to the next). Peters (1983) cited various authors who published equations that either support the grouping of all pelagic organisms under a single equation or instead stress differences among groups. He concluded that there are grounds for different opinions, especially since the same data were sometimes used to support opposing opinions (e.g. Eqs. $12-14$ vs Eq. 7).

Concerning $\tau_{\text {min }}$, using Eqs. (12)-(14) instead of Eq. (7), in conjunction with Eqs. (4) \& (5), leads to the following expressions:

$$
\begin{aligned}
& \tau_{\min }=990 L^{0.73} \text { (unicells) } \\
& \tau_{\min }=471 L^{0.70} \text { (poikilotherms) } \\
& \tau_{\min }=211 L^{0.70} \text { (homoiotherms) }
\end{aligned}
$$

When using Eqs. (12)-(14) instead of Eq. (7), the change in $\tau_{m n}$ is computed with Eq. (11) in cases of organisins feeding within their own group and with the following equations when an organism feeds upon another group:

$$
\tau_{\min 2} / \tau_{\min 1} \text { (poikilotherm/unicell) }
$$

$$
=(471 / 990)\left(L_{2}^{070} / L_{1}^{0.73}\right) \approx 0.5 \xi_{21}{ }^{070}
$$

and:

$$
\begin{aligned}
\tau_{\min 2} / \tau_{\min 1} \text { (homoiotherm/poikilotherm) } & \\
& =(211 / 471)\left(L_{2}{ }^{0.70} / L_{1}{ }^{070}\right) \approx 0.5 \xi_{21}{ }^{0.70}
\end{aligned}
$$

Given that homoiotherms seldom feed directly on unicells, using Eq. (10) instead of Eqs. (12)-(14) would cause, at maximum, an overestimation of $\tau_{\min 2} / \tau_{\text {nin: }}$ by a factor of 2 . In oceans, the usual $\xi_{21}$ of marine pelagic organisms to their food particles (ratio of ESDs) tend to cluster around a value of 14 (Sheldon et al. 1977) and can be as high as $4.8 \times 10^{4}(100 \mathrm{~mm}$ salp Cyclosalpa polae feeding on $2.1 \mu \mathrm{m}$ particles; Harbison \& McAlister 1979); these values correspond to $\xi_{21}{ }^{0.57}=6$ and 1370 , respectively. It follows that an overestimation of $\tau_{\min 2} / \tau_{\min 1}$ by a factor of 2 is small relative to the magnitude of $\xi_{21}{ }^{0.67}$ in the marine environment. Hence, for simplicity, Eq. (10) will be used in the remainder of the present paper. In oceans, the ratio $\tau_{\min 2} / \tau_{\min 1}$, which quantifies the lengthening of $\tau_{\text {min }}$ resulting from $L_{2}$-sized organisms feeding on $L_{1}$-sized prey, thus ranges from 1 to $1370\left(\xi_{21}=1\right.$ and $4.8 \times 10^{4}$, respectively, Eq. 10).

\section{Export of organic particles towards deep waters $\left(\tau_{s}\right)$}

There is no simple way to quantify the residence time of $\mathrm{BC}$ in deep waters. The significant point for cli- 
Fig. 1. Log-log plots and ordinary least-square (OLS) linear regressions (lines): (a) sinking velocity of faecal pellets $(S)$ as a function of their diameter $(D)($ Eq. 21): (b) $D$ as a function of the length $(L)$ of the organism producing them (Eq. 24); (C) $S$ as a function of $L$ (Eq. 26); (d) volume of faecal pellets $(P V)$ as a function of the dry carbon body mass (MC) of organisms producing them (Eq. 28). Transformations of $D$ and $L$ into $P V$ and $M C$, respectively, are explained in the text
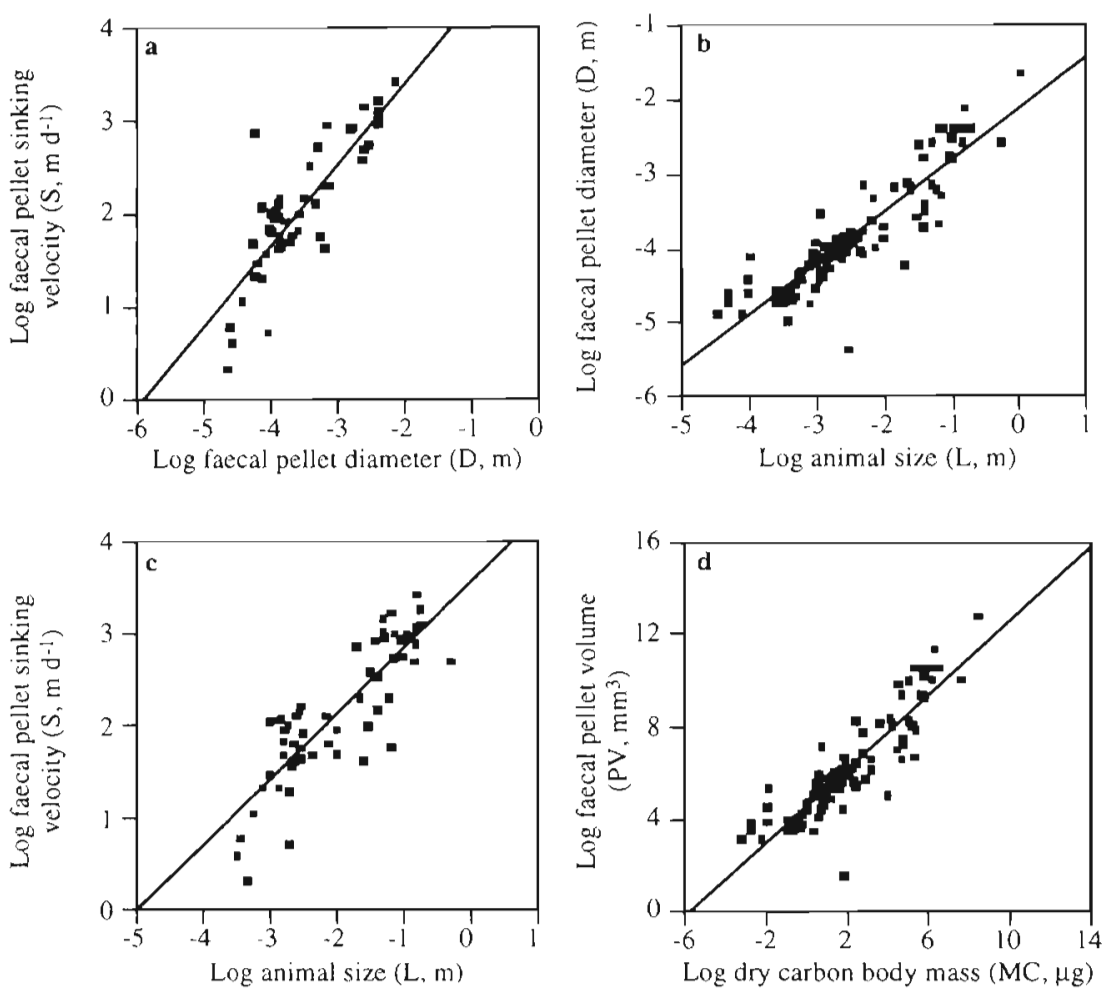

mate change, however, is how fast $\mathrm{BC}$ sinks out of the upper water column, i.e. how fast BC becomes sequestered. This characteristic is amenable to quantification using observations made in the upper water column. Sequestration occurs when BC reaches $z_{s}$, defined in the 'Introduction' as the depth from which it cannot rapidly return to the surface waters or the atmosphere.

The main mechanism responsible for the sedimentation of organic particles is their aggregation in larger, faster-sinking particles. In other words, it is considered here that living, healthy organisms generally do not sink or, if they do, they sink only slowly. In contrast, senescent or dead organisms tend to aggregate in large organic particles, which increases their sinking velocity. Sinking organic particles include marine snow (aggregates of various particles, including phytoplankton) and faecal pellets. The former result from physico-chemical aggregation of particles (e.g. Kiørboe 1993), whereas the latter are by-products of feeding activity. The type of food web determines the type of particles that are being aggregated into marine snow, but the formation and fate of marine snow are mostly controlled by hydrodynamics. Because the present paper focuses on direct food-web effects, only faecal pellets are considered below.

The approach to quantifying the potential sequestration of $\mathrm{BC}$ contained in sinking organic aggregates is to determine the residence time $\left(\tau_{s} ; d\right)$ of these particles above $z_{5}$ :

$$
\tau_{s}=z_{s} / S
$$

where $S$ is the sinking velocity of particles $\left(\mathrm{m} \mathrm{d}^{-1}\right)$. The following development first considers the estimation of $S$ from the diameter of faecal pellets $(D)$ and then from the size $(L)$ of organisms producing the pellets. The latter approach is especially useful when $D$ is not known.

Fig. 1a summarises published information on $S$ as a function of $D(\mathrm{~m}$; ESD). The following equation accounts for the general trend in the figure:

$$
\begin{gathered}
S=0.142 \times 10^{6} D^{0.87}(\mathrm{r}=0.86, \mathrm{n}=57, \mathrm{p}<0.001 ; \\
\text { OLS regression of } \log S \text { on } \log D)
\end{gathered}
$$

Using Eq. (21), the computation of $\tau_{s}$ (Eq. 20) is straightforward:

$\tau_{\mathrm{S}}=z_{\mathrm{s}} /\left(0.142 \times 10^{6} D^{087}\right)=7.042 \times 10^{-6} z_{\mathrm{s}} D^{-0.87}$

The change in $\tau$ that results from smaller organisms (size $L_{1}$ ) being aggregated in larger faecal pellets (size $D_{2}$ ) is computed as follows (Eqs. $10 \& 22$ ):

$$
\begin{aligned}
\tau_{\min 1} / \tau_{\mathrm{s} 2} & =\left(385 L_{1}{ }^{0.67}\right) /\left(7.042 \times 10^{-6} Z_{\mathrm{s}} D_{2}{ }^{-0.87}\right) \\
& =55 \times 10^{6} L_{1}^{0.67} D_{2}{ }^{0.87} / Z_{\mathrm{s}}
\end{aligned}
$$

In order to obtain ratios always $\geq 1$, the numerator in Eq. (23) is $\tau_{\min 1}$ (which was the denominator in 
$\left.\tau_{\min 2} / \tau_{\min 1}, E q .11\right)$. This is because the aggregation of $\mathrm{BC}$ in faecal pellets shortens $\tau_{\mathrm{s}}$, whereas the incorporation of $B C$ into larger organisms lengthens $\tau_{\text {min }}$ (see above). It must be noted that $\tau_{\mathrm{s} 2}$ is compared in Eq. (23) to the minimum turnover time of $B C$ prior to aggrega. tion $\left(\tau_{\min 1}\right)$, not to $\tau_{\mathrm{s} 1}$. This is because, as mentioned above, in the present study it is considered that living, healthy organisms generally do not sink, or sink slowly. Hence, $\tau_{\mathrm{s} 2}$ is compared not to $\tau_{\mathrm{s} 1}$ (which is theoretically infinite) but to $\tau_{\min 1}$.

Eq. (23) can be used in cases where the size of faecal pellets is known. In several instances, as already mentioned, in Eq. (22) it is more interesting and easier to replace the size of faecal pellets (D), which may be unknown, by the size of organisms $(L)$ producing the pellets. The resulting general relationship is (Fig. 1b)

$$
\begin{gathered}
D=7.301 \times 10^{-3} L^{0.69}(\mathrm{r}=0.89, \mathrm{n}=108, \mathrm{p}<0.001 ; \\
\text { OLS regression of } \log D \text { on } \log L)
\end{gathered}
$$

where $D$ and $L$ are both in metres. Combining Eqs. (21) and (24) allows one to compute $S$ from $L$ :

$$
S=1.965 \times 10^{3} L^{0.50}
$$

The above expression is not necessarily the same as the direct regression of $S$ on $L$. This is even more the case here because, as a consequence of missing information for several taxa, Regressions (21) \& (24) were not computed on exactly the same data sets. Direct regression of $S$ on $L$ gives (Fig. 1c)

$$
\begin{gathered}
S=3.650 \times 10^{3} L^{0.72}(\mathrm{r}=0.85, \mathrm{n}=58, \mathrm{p}<0.001 ; \\
\text { OLS regression of } \log S \text { on } \log L)
\end{gathered}
$$

Eq. (26), which is not very different from the previous one, will be used below. Because of the missing data, the number of points used to compute Regressions (21) \& (26) is smaller than for Regression (24).

Eq. (26) covers a wide range of taxa. It is nevertheless consistent with expressions derived for specific groups. For example, Uye \& Kaname (1994) published a general relationship for marine planktonic crustaceans in which the mean pellet volume $\left(P V_{i} \mu \mathrm{m}^{3}\right)$ is expressed as a function of individual dry carbon body mass of organisms ( $M C$; $\mu g$ ):

$$
\begin{gathered}
\log P V=4.56+0.85 \log M C(r=0.93, n=119 \\
\text { OLS regression })
\end{gathered}
$$

The equation is based on data for 10 copepod taxa and 1 mysiid (Neomysis japonica), each taxon being represented by several individuals. In order to compare Eq. (24) to Eq. (27), pellet diameters used for Eq. (24) were transformed into $P V\left[P V=0.524\left(D \times 10^{6}\right)^{3}\right.$, assuming spherical shapel and body lengths were transformed into $\mathrm{MC}\left[M=6.07\left(L \times 10^{6}\right)^{2.59}, \mathrm{Eq} \cdot(4)\right.$; dry mass $=0.1 \mathrm{M}$ and $\mathrm{MC}=0.4$ dry mass; Peters 1983 , his Appendix Ia; hence, $\left.\mathrm{MC}=0.24\left(L \times 10^{15.5}\right)\right]$. The resulting lin- ear regression is

$$
\begin{gathered}
\log P V=4.599+0.798 \log \mathrm{MC}(\mathrm{r}=0.90, \mathrm{n}=107, \\
\mathrm{p}<0.001 ; \text { OLS regression; Fig. } 1 \mathrm{~d})
\end{gathered}
$$

Eq. (28) is strikingly similar to Eq. (27). This indicates that considering a much wider range of taxa than did Uye \& Kaname (1994) $\left(10^{-1}\right.$ to $10^{3} \mu \mathrm{g} \mathrm{C}$ ind ${ }^{-1}$ in their paper versus $10^{-3}$ to $10^{9} \mu \mathrm{g} C$ ind.$^{-1}$ in the present study) does not fundamentally change the relationship between pellet and body sizes. As already shown for Eqs. (4) \& (10), allometric relationships over the whole range of marine pelagic organisms, although sometimes incorrect for individual taxa, provide useful tools for such studies as the present one.

Combining Eqs. (20) and (26) gives an expression for the residence time above depth $z_{\mathrm{s}}$ of $\mathrm{BC}$ incorporated in the faecal pellets of an $L$-sized organism $\left(\tau_{\mathrm{s}}\right)$, as a function of $L$ :

$$
\tau_{\mathrm{s}}=z_{\mathrm{s}} /\left(3.650 \times 10^{3} L^{0.72}\right)=2.740 \times 10^{-4} z_{\mathrm{s}} L^{-0.72}(29)
$$

The change in $\tau$ that results from $L_{1}$-sized organisms being aggregated in faecal pellets produced by $L_{2}$-sized organisms is computed as ioliows (Eqs. $i \hat{U} \&$ 29):

$$
\begin{aligned}
\tau_{\min } / \tau_{\mathrm{s} 2} & =\left(385 L_{1}^{067}\right) /\left(2.740 \times 10^{-4} Z_{\mathrm{s}} L_{2}^{-0.72}\right) \\
& =140 \times 10^{4} L_{1}^{0.67} L_{2}^{0.72} / Z_{\mathrm{s}}
\end{aligned}
$$

Eqs. (29) \& (30) are used instead of Eqs. (22) \& (23) when the size of faecal pellets is not known

Eq. (30) may be simplified as follows:

$$
\tau_{\min 1} / \tau_{\mathrm{s} 2} \approx 140 \times 10^{4}\left(L_{1} L_{2}\right)^{07} / Z_{\mathrm{s}}
$$

For $z_{\mathrm{s}}=1000 \mathrm{~m}$ (see above), the ratio $\tau_{\min 1} / \tau_{s 2}$, which quantifies the shortening of $\tau$ resulting from $L_{2}$-sized organisms feeding on $L_{1}$-sized living particles, ranges in oceans from $4 \times 10^{-6}$ to $6 \times 10^{2}$. These values correspond to $L_{1} L_{2}=6 \times 10^{-13} \mathrm{~m}^{2}(3 \mu \mathrm{m}$ flagellate Monosiga sp. feeding on $0.2 \mu \mathrm{m}$ heterotrophic bacteria; Fenchel 1982,1984 ) and $L_{1} L_{2}=0.3 \mathrm{~m}^{2}$ (30 $\mathrm{m}$ blue whale $\mathrm{Bal}$ aenoptera musculus feeding on $10 \mathrm{~mm}$ krill; Einarsson 1945, Barnes \& Creah 1988), respectively.

It is now well known that degradation of sinking faecal pellets may be very rapid. For example, the contribution of copepod faecal pellets to BC sequestration is generally quite small (references and discussion in Fortier et al. 1994). Hence, estimates of $\tau_{5}$ computed with either Eq. (22) or (29) are minimum values. The resulting underestimation of true $\tau_{\mathrm{s}}$ may be large for pellets that sink slowly, but it is quite small for pellets that sink fast. In oceans, the only faecal pellets that play a significant role in $\mathrm{BC}$ sequestration are those which sink very rapidly, such as those of salps, i.e. $S>$ 500 and even $>1000 \mathrm{m.} \mathrm{d}^{-1}$ (Wiebe et al. 1979, Caron et al. 1989, Drits et al. 1993), which correspond to $\tau_{s}<2$ and $<1 \mathrm{~d}$, respectively, for $z_{\mathrm{s}}=1000 \mathrm{~m}$. 


\section{Relationships between $\tau_{\min }$ and $\tau_{\mathrm{s}}$}

Using the above equations, it is possible to derive general relationships between $\tau_{\min }$ and $\tau_{\mathrm{s}}$, In the case of faecal pellets of known diameters, combining Eqs. (10) and (22) provides the relationship between $\tau_{\min 1}$ and $\tau_{\mathrm{s} 2}$ :

$$
\tau_{\min 1} \tau_{s 2}=385 L_{1}^{0.67} 7.042 \times 10^{-6} Z_{\mathrm{s}} D_{2}^{-0.87}
$$

where $L_{1}$ and $D_{2}$ are the diameters of the individual particles and pellets, respectively. Hence,

$$
\tau_{\min :} \tau_{\mathrm{s} 2}=2.7 \times 10^{-3} z_{\mathrm{s}} L_{1}^{0.67} D_{2}{ }^{-0.87}
$$

Taking $z_{\mathrm{s}}=1000 \mathrm{~m}$, Eq. (32) becomes

$$
\tau_{\mathrm{s} 2}=2.7 L_{1}{ }^{067} D_{2}{ }^{-0.87} / \tau_{\min 1}
$$

When particles are incorporated in faecal pellets, $\tau_{52}$ of $\mathrm{BC}$ in the resulting aggregates is inversely related to $\tau_{\min 1}$ of $B C$ in the individual particles, with a factor of ca $2.7 L_{1}^{0.7} D_{2}^{-0.9}$.

When the size of faecal pellets is not known, the relationship between $\tau_{\mathrm{mn} 1}$ of $\mathrm{BC}$ in the food particles and $\tau_{\mathrm{s} 2}$ of $\mathrm{BC}$ in pellets is obtained by combining Eqs. (10) and (29):

$$
\tau_{\min 1} \tau_{\mathrm{s} 2}=385 L_{1}^{0.67} 2.740 \times 10^{-4} Z_{\mathrm{s}} L_{2}^{-072}
$$

where $L_{1}$ and $L_{2}$ are the sizes of the food particles and pelagic organisms, respectively. Hence,

$$
\tau_{\min , 1} \tau_{\mathrm{s} 2} \approx 10^{-1} Z_{\mathrm{s}} L_{1}{ }^{0.67} L_{2}{ }^{-072}
$$

Taking $z_{\mathrm{s}}=1000 \mathrm{~m}$, Eq. 34 becomes

$$
\tau_{\mathrm{s} 2} \approx 100 L_{1}^{0.67} L_{2}^{-0.72 / \tau_{\min 1}}
$$

It follows that $\tau_{\mathrm{s} 2}$ of $\mathrm{BC}$ in faecal pellets is inversely related to $\tau_{\min 1}$ of $\mathrm{BC}$ in the food particles, with a factor of ca $100\left(L_{1} / L_{2}\right)^{0.7}$.

The relationship between the minimum turnover time of $\mathrm{BC}$ in the body mass of a pelagic organism ( $\left.\tau_{\min }\right)$ and the residence time of $B C$ in its faecal pellets $\left(\tau_{s}\right)$ is computed similarly to Eqs. (34) \& (35):

$$
\tau_{\text {min }} \tau_{\mathrm{s}} \approx 10^{-1} z_{\mathrm{s}} L^{0.67} L^{-0.72}
$$

Taking $z_{\mathrm{s}}=1000 \mathrm{~m}$, the above equation becomes

$$
\tau_{\text {mun }} \tau_{s} \approx 100 L^{0.67} L^{-0.72}
$$

Because $L^{0.67} L^{-0.72} \approx 1$, it follows that

$$
\tau_{\text {min }} \tau_{\mathrm{s}} \approx 100
$$

Hence,

$$
\tau_{\min } \approx 100 / \tau_{\mathrm{s}} \text { and } \tau_{\mathrm{s}} \approx 100 / \tau_{\min }
$$

In other words, when living particles are aggregated in faecal pellets, the $\tau_{\mathrm{s}}$ of $\mathrm{BC}$ in the resulting pellets is inversely related to $\tau_{\mathrm{min}}$ of $\mathrm{BC}$ in the pelagic organism, with a factor of ca 100 .
Aggregation of living particles by feeding activity has 2 consequences. On the one hand, Eq. (11) shows that feeding lengthens the residence time of $\mathrm{BC}$ incorporated in body masses $\left(\tau_{\min 2}>\tau_{\min 1}\right)$. This favours long-lived organic $C$, either by retention in the longlived pool of $\mathrm{BC}$ that was already there or by transfer to the long-lived pool of $\mathrm{BC}$ that would have been otherwise short-lived. On the other hand, Eqs. (32)-(36) show that $\tau_{\min 1}$ and $\tau_{\mathrm{s} 2}$ are inversely related. Hence, the production of faecal material may favour $\mathrm{BC}$ sequestration below $z_{\mathrm{s}}$ either by keeping among the fast-sinking material some particles that were already sinking fast or by transferring to sinking particles BC that would have been otherwise remineralized above $z_{\mathrm{s}}$.

\section{FUNCTIONAL GROUPS OF ORGANISMS BASED ON $\tau_{\text {min }}, \tau_{\mathrm{s}}$ AND $\xi$}

The minimum turnover time of $\mathrm{BC}\left(\tau_{\min }\right)$ and its residence time above $z_{\mathrm{s}}\left(\tau_{\mathrm{s}}\right)$ are 2 key criteria for establishing functional groups of organisms to be used in studies of pelagic food webs. By reference to the $3 \mathrm{BC}$ pools defined by Legendre \& Le Fèvre (1992) and discussed in the 'Introduction', the first important threshold is $\tau_{\min }=10^{-2} \mathrm{yr}=3 \mathrm{~d}$. The size corresponding to this threshold is $L=700 \mu \mathrm{m}$ (Eq. 10). Below $\tau_{\min }=3 \mathrm{~d}$, organic $\mathrm{C}$ is short lived and, above, it becomes long lived and may possibly be sequestered. Hence, organisms that transfer $\mathrm{BC}$ across the $\tau_{\mathrm{min}}=3 \mathrm{~d}$ threshold play an essential role in mediating water-column $\mathrm{BC}$ fluxes. Concerning sequestration, the main condition is rapid export below $Z_{\mathrm{S}}$ (1000 $\mathrm{m}$ here). The threshold for significant sequestration is taken here as being $\tau_{\mathrm{s}} \leq 2 \mathrm{~d}$. The corresponding size of organisms is $L=60 \mathrm{~mm}$ (Eq. 29).

Fig. 2 shows the positions of 139 taxa and stages of marine pelagic organisms in a scatter diagram of their $\tau_{\text {min }}$ versus the $\tau_{\min }$ of their food particles (Eq. 10). Because of Eq. (36), the abscissa is both the minimum turnover time of $\mathrm{BC}$ in organisms $\left(\tau_{\operatorname{mn} 2}\right)$ and the residence time of $\mathrm{BC}$ in their sinking faecal pellets $\left(\tau_{\mathrm{s}}\right)$. Threshold $\tau_{\min }=3 \mathrm{~d}$ is drawn on the 2 axes and threshold $\tau_{\mathrm{s} 2}=2 \mathrm{~d}$ is drawn on the abscissa (solid lines). The combined thresholds delineate 4 broad groups of organisms, whose taxonomic composition is detailed in Fig. 3. Group 1 (Fig. 3a) consists of microzooplankton (flagellates, ciliates-including tintinnids - and copepod nauplii). These organisms feed on prey with $\tau_{\min }<3 \mathrm{~d}$ and themselves have $T_{\text {min }}<3 \mathrm{~d}$. Hence, they are efficient at remineralizing BC. Group 2 (Fig. 3b) includes mesozooplankton, most macrozooplankton, and fish larvae. These are characterized by $\tau_{\min }>3 \mathrm{~d}$, they feed on prey whose 


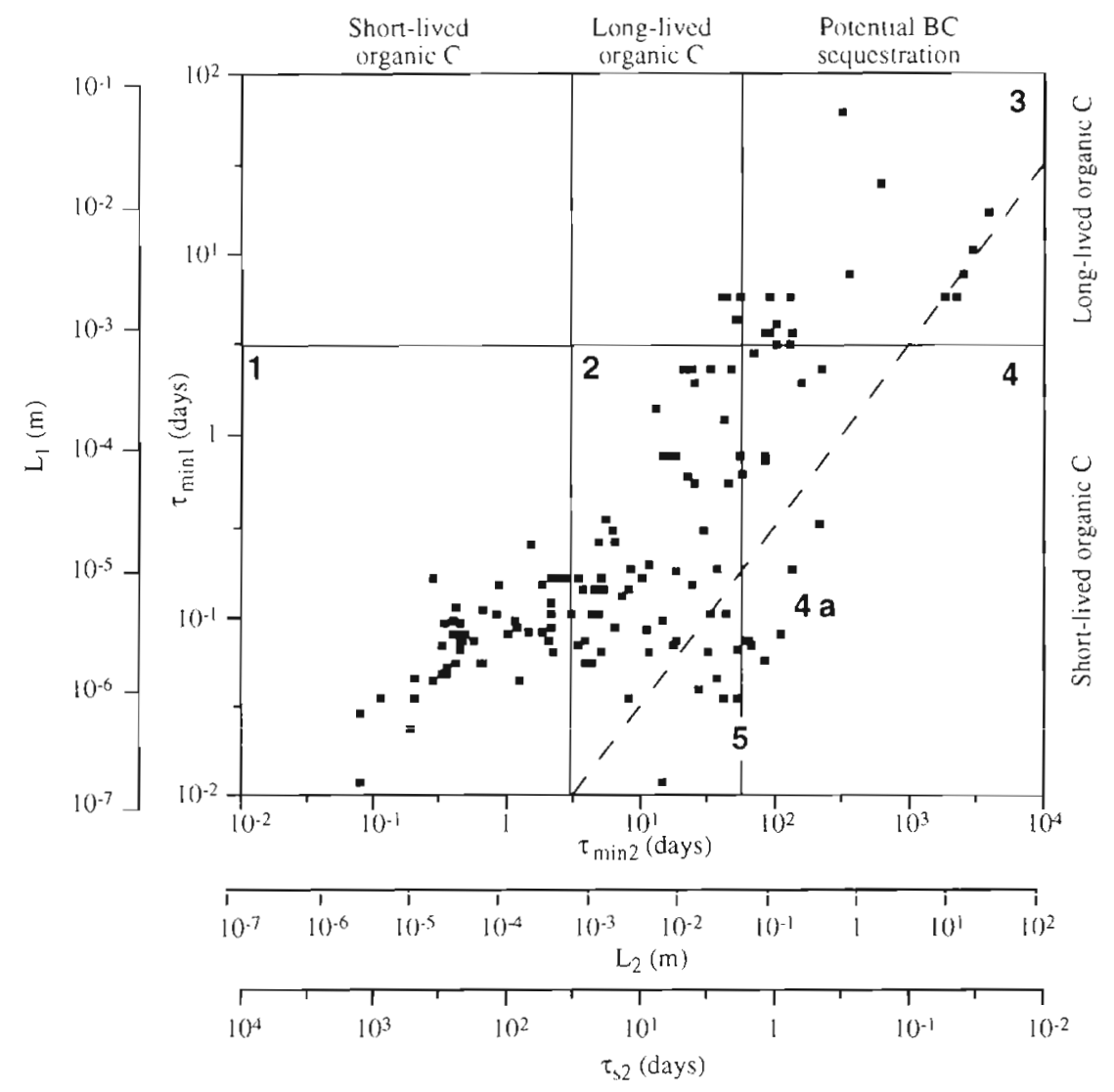

Fig. 2. Scatter diagram of 139 taxa and developmental stages of marine pelagic organisms. $\tau_{\operatorname{mnn} 2}$ : minimum turnover time of $\mathrm{BC}$ in organisms as computed from their length ( $L_{2} ;$ Eq. 10$)$. $\tau_{s 2}$ : calculated residence time of $B C$ in sinking faecal pellets of $L_{2}$-sized organisms with $z_{\mathrm{s}}=1000 \mathrm{~m}$ (Eq. 29 or 36). $\mathrm{T}_{\min 1}$ : minimum turnover time of $\mathrm{BC}$ in food organisms as computed from their length $\left(L_{1}\right.$; Eq. 1.0). Top and right: carbon pools corresponding to $\tau$, as defined by Legendre \& Le Fèvre (1992). Solid lines: threshold values $\tau_{\min }=3 \mathrm{~d}(<3 \mathrm{~d}$ : shortlived organic $C$ ) and $\tau_{\mathrm{s} 2}=2 \mathrm{~d}(>2 \mathrm{~d}$ : potential $\mathrm{BC}$ sequestration). Dashed line: $\xi_{21}=L_{2} / L_{1}=5 \times 10^{3}$ (to the right: large microphagous zooplankton). Identified on the figure: 4 main functional groups of taxa and stages ( 1 to 4 ) and 2 additional groups ( $4 \mathrm{a}$ and 5 ) (see text) $\tau_{\min }<3 \mathrm{~d}$, and they produce faecal material that sinks relatively slowly. Hence, they transfer $\mathrm{BC}$ above the $\tau_{\min }=3 \mathrm{~d}$ threshold, but they do not contribute to $\mathrm{C}$ sequestration. Group 3 (Fig. 3c) comprises most fish and all marine mammals. These have $\tau_{\text {min }}>3 \mathrm{~d}$, feed on prey whose $\tau_{\min }>3 \mathrm{~d}$, and produce faeces that often sink fast $\left(S \geq 500 \mathrm{~m} \mathrm{~d}^{-1}\right.$; Bray et al. 1981, Robinson \& Bailey 1981; hence, $\tau_{s} \leq 2 \mathrm{~d}$ ). It follows that they do not transfer $\mathrm{BC}$ across the $\tau_{\min }=3 \mathrm{~d}$ threshold, but some taxa may contribute to $C$ sequestration. Group 4 (Fig. 3d) consists of a few macrozooplankton organisms with $\tau_{\min }>3 \mathrm{~d}$, which feed on prey that include taxa whose $\tau_{\min }<3 \mathrm{~d}$ and produce faecal material that sinks fast. Hence, they transfer $\mathrm{BC}$ above the $\tau_{\min }=3 \mathrm{~d}$ threshold, and they may also contribute to C sequestration.

In order to further refine the above functional groups of taxa, one can use as additional criterion the size ratio of consumers to their food particles $\left(\xi_{21}=L_{2} / L_{1}\right)$. Fortier et al. (1994) recognised that large marine planktonic microphages $\left(\xi_{21}>5 \times 10^{3}\right)$ are most efficient at repackaging small particles into larger ones. Accordingly, the threshold $\xi_{21}=5 \times 10^{3}$ in drawn on Fig. 2 (dashed line). Below this threshold, a group of large microphagous zooplankton can be distinguished either from within Group 4 (designated as Group 4 a in Fig. 2), or across Groups 2 and 4 (designated as Group 5 in Fig. 2). Groups 4 a and 5 consist of organisms with such feeding behaviour that they can capture food particles almost 4 orders of magnitude smaller than themselves. Hence, these organisms may be treated separately in food-web models.

The above approach uses a priori $\tau_{m, n}, \tau_{s}$, and $\xi$ criteria to define functional groups of organisms. In the present study, the criteria were $\tau_{\min }=3 \mathrm{~d}, \tau_{s}=2 \mathrm{~d}$, and $\xi_{21}=$ $5 \times 10^{3}$ (Figs. $2 \& 3$ ). In other studies, slightly different values could be used, which would produce somewhat different groups of taxa. Equations in the present paper describe functional relationships between, on the one hand, $L_{1}$ and $\tau_{\min 1}$ and, on the other hand, $L_{2}$, $\tau_{\min 2}$ and $\tau_{52}$. With these relationships, it becomes possible to use the length of any organism for computing 2 critical characteristics of $\mathrm{BC}$ in the marine environment, i.e. the minimum turnover time of $B C$ in the organism and the residence time of BC above $z_{\mathrm{s}}$. Using our approach, any organism can be positioned in Fig 2, the functional group to which it belongs determined, and its contribution to the channelling of $\mathrm{BC}$ towards the short-lived, long-lived and sequestered pools assessed. 


\begin{tabular}{|lr|}
\hline Mammals & 1 \\
Fish & 2 \\
Fish larvae & 3 \\
Salps & 4 \\
Pteropods & 5 \\
Euphausiids & 6 \\
Copepods & 7 \\
Unmature copepods & 8 \\
Other crustaceans & 9 \\
Tintinnids & 10 \\
Ciliates & 11 \\
Flagellates & 12 \\
Others & 13 \\
\hline
\end{tabular}

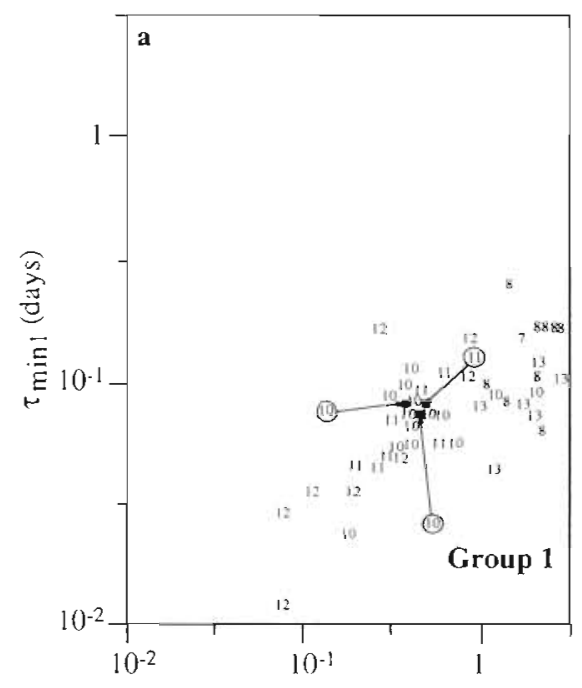

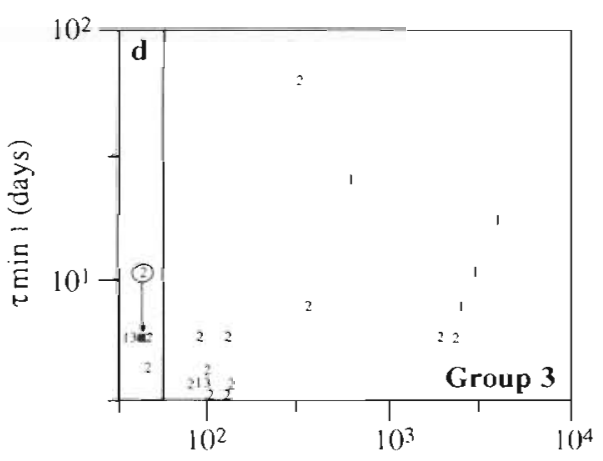

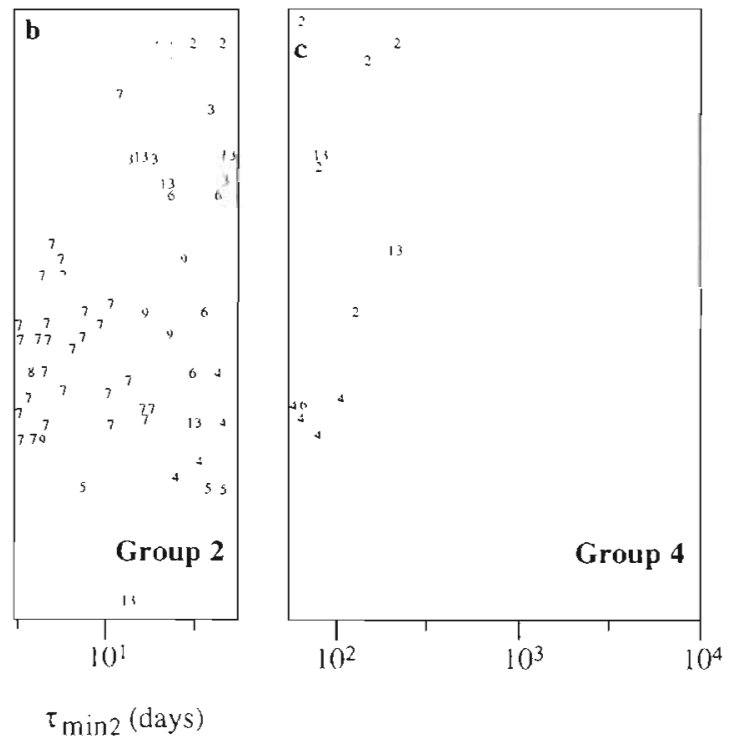

Fig. 3. Detailed taxonomic compositions of Groups 1 to 4 identified on Fig. 2. (a) Group 1: 48 microzooplankton taxa and stages, (b) Group 2: 59 mesozooplankton, macrozooplankton, and fish larval taxa and stages, (c) Group 3: 20 fish and marine mammal taxa (including 1 chaetognath and 3 fish taxa plotted to the left of $\tau_{\mathrm{s} 2}=2 \mathrm{~d}$ in Fig. 2), (d) Group $4: 12$ macrozooplankton taxa

\section{FOOD-WEB REGULATION OF BIOGENIC CARBON FLUX}

Our study shows that general allometric relationships can be derived for the whole size range of marine pelagic organisms. The approach was applied to the following sets of variables: (1) body length of organisms $(L)$ as a function of wet body mass (M) (Eq. 4); (2) change in minimum turnover time of $B C\left(\tau_{\min }\right)$ that results from smaller organisms $\left(L_{1}\right)$ being eaten by larger ones $\left(L_{2}\right)\left(\tau_{\min 2} / \tau_{\min 1}\right)$, given as a function of the size ratio of the organisms $\left(\xi_{21}=L_{2} / L_{1}\right)$ (Eq. 11); (3) size of faecal pellets $\left(D_{2}\right)$ as a function of the size of organisms producing them $\left(L_{2}\right)$ (Eqs, $24 \& 28$ ); (4) residence time above depth $z_{\mathrm{s}}$ of $\mathrm{BC}\left(\tau_{\mathrm{s} 2}\right)$ either in $D_{2}$-sized faecal pellets, expressed as a function of $D_{2}$ (Eq. 22), or in pellets produced by an $L_{2}$-sized organism, given as a function of $L_{2}$ (Eq. 29); (5) change in $\tau\left(\tau_{\min 1} / \tau_{s 2}\right)$ that results from $L_{1}$-sized organisms being either aggregated in
$D_{2}$-sized faecal pellets, expressed as a function of $z_{\mathrm{s}}, L_{1}$, and $D_{2}$ (Eq. 23), or aggregated in the pellets of $L_{2}$-sized organisms, given as a function of $z_{5}, L_{1}$, and $L_{2}$ (Eqs. 30 $\& 31$ ); (6) relationship between $\tau_{\min 1}$ and $\tau_{\mathrm{s} 2}$, involving the sizes of individual particles $\left(L_{1}\right)$ and those of either faecal pellets $\left(\mathrm{D}_{2}\right)$ (Eq. 33) or pelagic organisms producing the pellets ( $\left.L_{2}\right)$ (Eq. 35); and (7) relationship between the minimum turnover time of $\mathrm{BC}$ in the body mass of a pelagic organism $\left(\tau_{\min 2}\right)$ and the residence time of $\mathrm{BC}$ in its faecal pellets $\left(\tau_{\mathrm{s} 2}\right)$ (Eq. 36). Discussion of some of the equations showed that, even if values computed for individual taxa or taxonomic groups were sometimes incorrect, results were satisfactory over the size range of marine pelagic organisms.

The above relationships can be used to assess foodweb regulation of the $\mathrm{BC}$ flux in oceans. On the one hand, Eq. (11) shows that incorporation of BC belonging to $L_{1}$-sized living particles in the body masses of $L_{2}$-sized organisms results in a lengthening of $\tau_{\min 2}$ rel- 


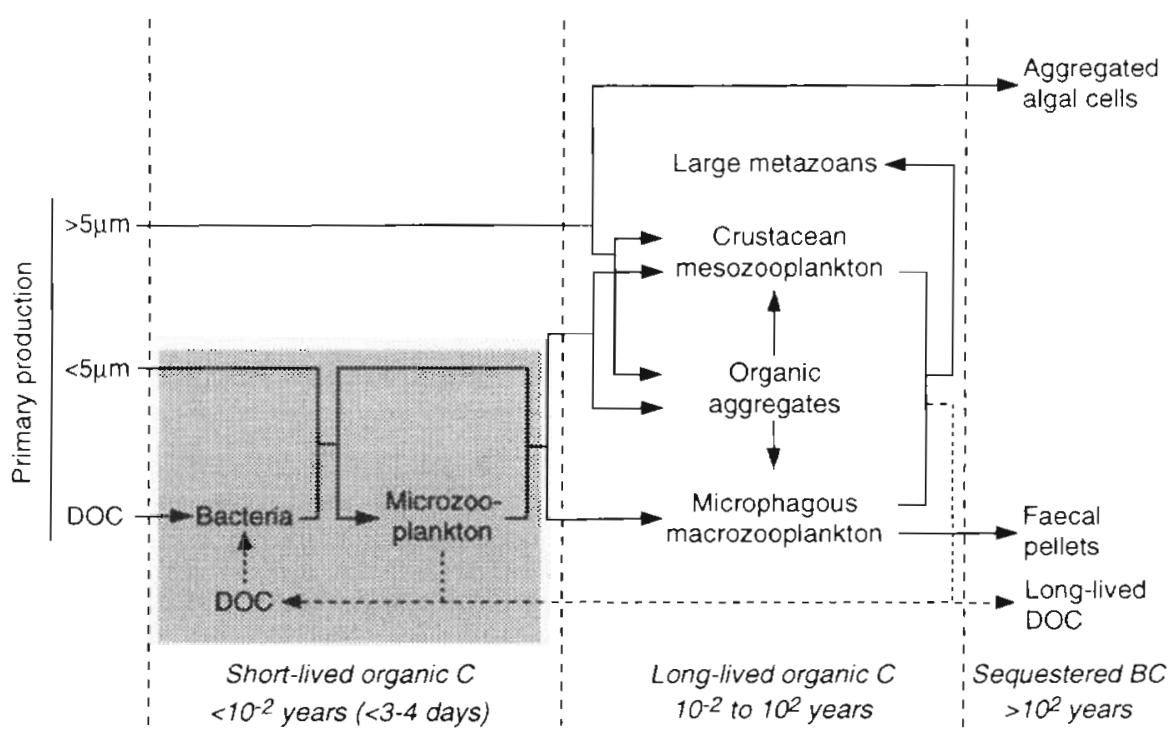

Fig. 4. Conceptual food-web mediated C flux model modified from Legendre (1996) and Legendre \& Rassoulzadegan (1996). Solid arrows: major flows of biogenic carbon in the euphotic zone of oceans, from 3 size classes of net (vs gross) primary production (cells $>5 \mu \mathrm{m}$, cells $<5 \mu \mathrm{m}$, and DOC) to 3 carbon pools (short-lived, long-lived, and sequestered, Legendre \& Le Fèvre 1992). Dashed arrows: food-web recycling of DOC, from consumers to heterotrophic bacteria (as consequence of viral lysis, sloppy feeding excretion by herbivores, and degradation of faeral material and other detritus; e.g. Kirchman ot al 19o3) and carbon sequestration as long-lived DOC. The shaded rectangle delineates the microbial food web

ative to $\tau_{\min 1}$ in proportion to $\xi_{21}=L_{2} / L_{1}$. This increases the flux towards the pool of long-lived organic carbon. On the other hand, Eqs. (30) \& (31) show that incorporation of $\mathrm{BC}$ from $L_{1}$-sized living particles in the faecal pellets of $L_{2}$-sized organisms results in a shortening of $\tau_{s 2}$ relative to $\tau_{\text {mn } 1}$ in proportion to $L_{1} L_{2}$. This increases the flux towards the pool of sequestered BC. It follows that, as the size $\left(L_{2}\right)$ of the organism feeding on $L_{1}$-sized prey increases, there is a proportional lengthening of $\tau_{\min 2}$ relative to $\tau_{\min 1}$ and proportional shortening of $\tau_{\mathrm{s} 2}$ relative to $\tau_{\min 1}$. The resulting enhanced flux towards the pools of long-lived organic $C$ and sequestered $\mathrm{BC}$ is significant for renewable resources and climate change, respectively.

When marine pelagic food webs are modelled, organisms are generally grouped into functional categories. In some studies, the groups are based on trophic position (e.g. Fasham et al. 1990) and, in others, on some combination of size and trophic position (e.g. Moloney \& Field 1991). Our study shows that the trophic position and size approaches can be combined in a general scheme, which is illustrated in Fig. 2. In order to do this, critical values (thresholds) must be set for $\tau_{\min }$ and $\tau_{5}$, and also $\xi_{21}$. Combining the first 2 criteria (plus eventually the third one) determines functional groups of taxa that reflect both trophic relationships and sizes and are pertinent to BC flux towards large organisms and deep waters. As mentioned above, the threshold values in the present paper $\left(\tau_{\min }=\right.$ $3 \mathrm{~d}_{1} \tau_{\mathrm{s}}=2 \mathrm{~d}$, and $\xi_{21}=5 \times 10^{3}$ ) could be modified to accommodate specific requirements. Fig. 2 can also be used to compare pelagic ecosystems, or to characterize changes (e.g seasonal) within ecosystems.

It is interesting to note that Fig. 2 rank-orders the functional groups of taxa in the almost exact same way as in the conceptual food-web mediated $C$ flux model of Legendre (1996) and Legendre \& Rassoulzadegan (1996), which is reproduced in Fig. 4. Group 1 corresponds to the microzooplankton compartment, which is part of the microbial food web. Group 2 covers crustacean mesozooplankton, some microphagous macrozooplankton, and larval fish (link towards large metazoans). Group 3 consists of large metazoans. Group 4 includes those microphagous macrozooplankton. whose fast-sinking faecal pellets contribute to $C$ sequestration. A major difference between Fig 2 and Fig. 4 is that the former does not consider deep DOC export or sinking phytoplankton aggregates because, as mentioned earlier, these important fluxes are not ultimately determined by food-web activity but instead by hydrodynamics. The agreement between the 2 figures supports the idea that functional groups based on the $\tau_{\text {mini }} \tau_{\mathrm{s}}$ and $\xi$ criteria are well suited for studying food-web mediated $C$ flux in oceans.

Acknowledgements. The authors thank P. Legendre, R. B. Rivkin, A. F. Vézina, and 3 anonymous reviewers for useful comments and suggestions. A research grant from the Natural Sciences and Engineerng Research Council of Canada and a Killam Research Fellowship (Canadd Council) to L.L. were instrumental in the completion of the work. This study is 
a contribution to the programme of GIROQ (Groupe interuniversitaire de recherches océanographiques du Québec).

\section{LITERATURE CITED}

Barnes LG, Creah C (1988) Kinds of whales. In: Harrison R, Bryden MM (eds) Whales, dolphins and porpoises. Fact on file publications, New York, p 24-43

Blueweiss L, Fox H, Kudzma V, Nakashima D, Peters R, Sams S (1978) Relationships between body size and some life history parameters. Oecologia 37:257-272

Bray RN, Miller AC, Geesey GG (1981) The fish connection: a trophic link between planktonic and rocky reef communities? Science 214:204-206

Caron DA, Madin LP, Cole JJ (1989) Composition and degradation of salp fecal pellets: implications for vertical flux in oceanic environments. J Mar Res 47:829-850

Drits AV, Arashkevich EG, Semenova TN (1993) Role of Pyrosoma, Salpae, and Copepoda in utilization and flux of organic matter off west Africa. Russ J Aquat Ecol 2:1-12

Einarsson H (1945) Euphausiacea I. Northern Atlantic species. Dana Report 27. AF Host and Son, Copenhagen

Fasham MJR, Ducklow HW, McKelvie DS (1990) A nitrogenbased model of plankton dynamics in the oceanic mixed layer. J Mar Res 48:591-639

Fenchel $T$ (1974) Intrinsic rate of natural increase: the relationship with body size. Oecologia 14:317-326

Fenchel T (1982) Ecology of heterotrophic microflagellates. I. Some important forms and their functional morphology. Mar Ecol Prog Ser 8:211-223

Fenchel T (1984) Suspended marine bacteria as a food source. In: Fasham MJR (ed) Flows of energy and materials in marine ecosystems. Theory and practice. Plenum Press, New York, p 301-315

Fortier L, Le Fèvre J, Legendre L (1994) Export of biogenic carbon to fish and to the deep ocean: the role of large planktonic microphages. J Plankton Res 16:809-839

Harbison GR, MCAlister VL (1979) The filter-feeding rates and

Editorial responsibility: Otto Kinne (Editor),

Oldendorf/Luhe, Germany particle retention efficiencies of three species of Clyclosalpa (Tunicata, Thaliacea). Limnol Oceanogr 24:875-892

Kiorboe T (1993) Turbulence, phytopankton cell size, and the structure of pelagic food webs. Adv Mar Biol 29:1-72

Kirchman DL, Lancelot C, Fasham MJR, Legendre L, Radach L, Scott M (1993) Dissolved organic matter in biogeochemical models of the ocean. In: Evans GT, Fasham MIJR (eds) Toward a model of ocean biogeochemical processes. Springer-Verlag, Berlin, p 209-225

Legendre L (1996) The biological $\mathrm{CO}_{2}$ pump in seasonally icecovered waters. Proc NIPR Symp Polar Biol 9:61-74

Legendre L, Le Fèvre J (1992) Interactions between hydrodynamics and pelagic ecosystems: relevance to resource exploitation and climate change. S Afr J Mar Sci 12:477-486

Legendre L, Rassoulzadegan F (1996) Food-web mediated export of biogenic carbon in oceans: environmental control. Mar Ecol Prog Ser 145:179-193

McArdle BH (1988) The structural relationship: regression in biology. Can J Zool 66:2329-2339

Moloney CL, Field JG (1991) The size-based dynamics of plankton food webs. I. A simulation model of carbon and nitrogen flows. J Plankton Res 13:1003-1038

Peters H (1983) The biological implication of body size. Cambridge Univ Press, Cambridge

Robinson BH, Bailey TG (1981) Sinking rates and dissolution of midwater fish fecal matter. Mar Biol 65:135-142

Sheldon RW, Sutcliffe WHJ, Paranjape MA (1977) Structure of pelagic food chain and relationship between plankton and fish production. J Fish Res Bd Can 43:2344-2353

Sokal RR, Rohlf FJ (1995) Biometry. The principles and practice of statistics in biological research. WH Freeman \& Co, New York

Uye SI, Kaname K (1994) Relations between pellet volume and body size for major zooplankters of the Inland Sea of Japan. J Oceanogr 50:43-49

Wiebe PH, Madin LP, Haury LR, Harbison GR, Philbin LM (1979) Diel vertical migration by Salpa aspera and its potential for large-scale particulate organic matter transport to the deep-sea. Mar Biol 53:249-255

Submitted: July 15, 1997; Accepted: January 7, 1998

Proofs received from author(s): March 13, 1998 